\title{
A VIDEO HOW-TO FOR BEGINNERS
}

The viewing of commercially produced television programs makes video production seem simple. Local productions may at first seem disappointing after viewers have been bombarded with sophisticated multicamera operations in commercial productions. It is, therefore, important to prepare teachers, students, and administrators for the results produced by small system, single camera activities. Fortunately, creativity is not restricted by the number of cameras available or by the necessity for state-ofthe-art equipment. Student enthusiasm for local productions will grow quickly after they have been allowed the chance to operate cameras and recorders.

The novice may be initially overwhelmed by the "simple" task of acquiring techniques and skills for utilizing equipment in the classroom; particularly if he/she is seeing that equipment for the first time. It makes little difference whether the camera is state-of-the-art or an old tube model; the control mechanisms will probably seem overwhelmingly complicated. This typical reaction may be obviated by the use of educational learning principles. Expertise, at least at a comfortable working level, may be acquired by using such techniques as simplifying and isolating key operating features with color codes or numbers and by having spaced practice of the psychomotor skills.

The emphasis in this article is, 1) to present an overview of how video works, 2) to show a step by step mechanical procedure, and 3) to give some suggestions for managing the operation. The general bibliography contains reference on the value of video to foreign language students, the use of broadcast television from satellites as a classroom compliment, and a list of video textbooks.

\section{The Camera}

The two main components of the camera are the lens and the picture tube (in the case of newer camers, the charge coupled device--CCD). Lens Light enters the camera through the lens. Two lenses will be all that are needed by most beginners. A wide angle lens, which produces broad pictures, and a telephoto zoom lens, which can give close ups, middle range, or distance shots. The image and the distance is controlled by a turning movement on the lens. Practice with the lens is essential for smooth focusing and adjustment of the iris/t-stops. The amount of light coming into the camera is controlled by turning the iris ring; the f-stops open up or close down, admitting more or less light. F-stop numbers are 22, 18, 11, 9, 5.6, $4.5,3$ and they indicate the size of the iris. The larger the number, the smaller the opening that admits light. After focusing it may be necessary to "stop down" by turning the f-stop ring to a larger number. To focus with a zoom lens, open the f-stops from 0 to 2, turn the zoom ring on the lens to the far 
right to reach the maximum close-up setting, adjust the focus ring, then turn the zoom ring back until the area wanted in the composition appears in the viewfinder. As long as the subject and the camera stay in the same general area, the picture will be in focus. If either moves, it will be necessary to refocus.

Light passes through the lens and the electrons are absorbed on a photoelectric surface. At the other end of the tube, a scanning motion reads the back of the photoelectric surface to create an electronic reaction to the spaces left by the absorbed electrons. The greater the amount of light that hits the front of the tube, the stronger the electronic reaction that finally produces the picture. The scanning motion is similar to the way one reads a printed page; from the upper left-hand corner horizontally across the printed line, jumping diagonally down to the beginning of the next line, finishing one colume and going to the next--creating a sync pulse.

Video pictures are made up of two fields--odd and even. To produce a picture the camera must read the two fields, first scanning the odd field and then scanning the even field. The viewer of a television picture sees a combination of the two fields. Without this combination there would be no picture just as is the case with weaving where there is no cloth without a warp and a woof. This process in the video camera thus converts reflected light from real objects into a series of electronic impulses (video signals) which are fed into a video recorder.

\section{The Video Cassette Recorder (VCB)}

The VCR converts the video signals from the camera, the electronic impulses, into magnetic impulses which are then recorded on the tape. In most VCR's the tape passes over two video heads at a slight angle so that the video information is recorded as long slanting lines called "helical scan" space at the top and bottom for the audio and control tracks. Each video head records or plays back one field (the off and even lines of scanning), each time it touches the tape. In addition to the scanning, the videotape contains the audio track and a sync track which allows the material to be machine-readable. The wrong sync signal is usually the problem when one tape will not play back correctly on another machine. A machine that needs adjusting, playback and record heads that need cleaning or alignment are maintenance factors that could cause problems when schools share videotapes.

\section{Beception}

Most television signals are broadcast as radio frequency (RF) signals. These signals are either transmitted through the air (from TV stations or from satellites) or through TV cable systems. The typical home TV set, a receiver, can only accept RF signals either through the antenna terminals or through an RF jack. TV monitors, on the other hand, do not have a tuner and can only handle video signals--from a VCR or other source. The video signal will 
only handle video signals--from a VCR or other source. The video signal will generally give a better image than $\mathrm{RF}$ signal so it is advantageous to use a monitor when you are, for example, playing a tape on your VCR. A monitor/ receiver has both RF and video signals, but is usually more expensive than the monitor or the receiver. Many schools provide for a central distribution system that provides RF signals to a number of TV receivers which can be located in any rooms or buildings that are wired into the system.

The TV monitor/receiver recreates the pictures in an inverse manner from how the camera operates. The magnetic impulses are converted to electronic impulses and then transmitted to the TV where the impulses become light, i.e., an image. The picture tube of the TV, acting as a reverse camera, with its gun shooting electrons onto the photoelectronic surface, line by line, coordinated by the sync pulse. Light and dark dots (about 500 per line) reflect how light entered the camera from with through shadows of gray to black to provide the image on the screen.

\section{Sound}

Sight and sound are equally important aspects of most television. Audio pick-up is dependent on the correct placement of microphones and the quality of those microphones.

Many cameras have microphones built into the units: as a rule, these mics are not good enough for most production work. Since they are housed in the camera case, they tend to amplify all noises associated with the camera's operation. External microphones plugged into the VCR generally provide better sound reproduction. The quality of the sound and the placement of the microphones can best be determined by monitoring the output with a pair of headphones plugged into the VCR. The same principles that apply to audio cassette recordings apply to the sound quality of video recordings.

\section{Composition}

Composition is a very important element of video production. Only by practicing and by observing other video programs can skill in composition be developed. Experience in still photography is an advantage in learning video composition. It is also important to read articles and books pertaining to this subject--some suggested readings are included in the bibliography. Depth of field (the clarity of elements within the composition) is manipulated by f-stops and by the zooming mechanism of the camera. It is very important to develop skill with lighting techniques to improve picture quality, establish mood, intensity, and depth. Too much light will wash out a picture and fade the scene, too little light will result in a dim picture and a loss of detail. Extra lighting in a classroom or other setting usually results in an improved picture. Teachers and students must learn to judge the scene by playing back test runs on a monitor to confirm what the eye has missed and to see if there is 
too much or too little light. The lighting terms "key" (primary), "back" (rim), and "fill" (side) have the same application in video as they do in photography.

\section{Becording}

Directions such as those listed below will help to organize students as they make their recording:

1. Connect camera cable to camera input on switch box or on the VCR deck.

2. Connect power sources to all machines.

3. Connect external microphones to the mic input of the VCR or the camera. Remember to insert the battery for wireless microphones.

4. Remove lens cap.

5. Insert cassette and depress the record button on the VCR.

6. Evaluate the image in the view-finder; adjust zoom, frame, and focus; adjust the iris for the correct light.

7. Monitor the audio on the headphones.

8. Set all index meters to zeroes.

9. Start recording via the trigger on the camera or by depressing play/ record on the VCR; run with the iris closed for five seconds and then open it up slowly for a test run of 3 to 5 minutes duration.

10. Stop the VCR, rewind the tape, playback to check on the quality of the lighting and the sound level; make any needed corrections and get ready to videotape again.

11. Additional tips:

a. Practice and keep notes

b. Never point the lens at a bright light (even when camera is turned off)

c. Keep the volume down on your TV monitor to reduce feedback.

d. Film a title for your tape and immediately label the tape providing all pertinent information--date, recording length, title, etc.

e. Remove the red button or break the tab on the cassette so that it may not be accidentally erased.

f. Save information on the speaker, the camera operator, speaker permission forms, etc.

g. Seek articles and books which will expand yourknowledge of video production.

\section{Erequent Problems}

1. When videotaping a class or a lecture the students' questions are not picked up by the microphone used by the speaker and these questions will not be understood by persons viewing the tape at a later date.

All student questions should be repeated or paraphrased by the speaker. 
2. The videotape is dark and the images are difficult to see.

Supplementary lighting is usually necessary for classrooms and auditoriums--invest in portable lighting kits; overhead projectors also can be an emergency lighting source.

3. The speaker's voice fades in and out.

The problem is usually caused by the speaker's movement from the fixed microphone to use visuals or to write on a blackboard. Put a lavalier microphone on the speaker.

4. Tapes are misplaced.

Label both the videocassette and its container. Film the title of the presentation using the speaker's name and objective--this can either be at the beginning or the end of the program.

5. Too much camera movement, zooming, or refocusing creates a sense of vertigo while watching the picture.

Give the student operators time to practice--if possible prepare them for any movement by the speaker. Have the operators watch the finished product so that they may profit from their mistakes.

\section{Terminology}

You should become familiar with the terminology associated with video. The following lists may give you some idea of the terms you should be able to recognize.

1. Types of connectors--bnc, rca, mini phone plugs, phone plugs, xir, and cannon plugs.

2. Video terms--coaxial, dolly, pan, tilt, break-out box, dubs, adapters, vhf, uhf.

\section{Video Formats and Standards}

Two problems that often confront the video user are the existence of different video formats and of different television/recording standards. Format refers to the type of device on which the recording is made and standards refer to the different television recording methods which are used in various countries.

Eormats Videotape (reel) formats include 1/2" EIAJ, 1-inch, and 2-inch quad.

There are three common videocassette formats--3/4" U-Matic, 1/2" VHS, and 1/2" Beta; a fourth format, $8 \mathrm{~mm}$ is popular for camcorders.

There are three primary international standards that are used in video recording and transmission:

NTSC (National Television Systems Committee) 525 lines

PAL (Phrase Alternate Line) 625 lines

SECAM (Sequential Color and Memory) 625 lines

Both PAL and SECAM give sharper pictures because they have more lines. 
None of these standards is compatible; the tapes will fit in machines from other countries (VHS is the same worldwide) but they will not play back correctly. There are two ways to deal with this problem: 1) use a multistandard VCR and monitor, 2) have the tapes converted by a device called a standards converter--these are presently very expensive and the commercial rates for conversion are, therefore, quite high.

Another standard that is being developed is High Definition TV (HDTV). Some technologists suggest that future receivers using digital applications will be able to have pictures of 900 lines. NHK of Japan has had a workable 1125 line system (based on an analogue system) since 1968, but digital systems will be more economical in bandwidth and in accuracy.

The forenunners for a common standard for color signals in 1983 were Britain's MAC and stretched MAC. It will, however, be quite some time before we see a solution to the problems of television standards.

\section{Suggested Readings}

Burrows and Wood. Television Production: Disciplines and Techniques. Dubuque, IA: Wm. C. Brown, Publishers, 1978.

Minor, Ed and Harvey Frye. Techniques for Producing Visual Instructional Media. New York: McGraw Hill, 1977.

Schmid, William T. Media Center Management, A practical Guide. New York, Hasting House.

Utz, Peter. The Complete Video Book. Englewood Cliffs, NJ: Prentice Hall, 1984.

---. Do-lt-Yourself-Video. Englewood Cliffs, NJ: Prentice Hall, 1984.

---. Video User's Handbook. Englewood Cliffs, NJ: Prentice Hall, 1984. Zettl, Herbert, Television Production-Handbook. Belmont, CA: Wadsworth Publishing Company, 1976.

Video PO Box 52255, Boulder, CO 80321-2255: monthly issues cover equipment news, buying guides, and videocassette reviews.

Video Review PO Box 919, Farmingdale, NY, 11737-9619: monthly issues include equipment news, equipment test reports, videocassette reviews.

Global Satellite Communications for Learning Worldwide (SCOLA) Creighton University, Telecommunications, California at 24th St., Omaha, NE, 68178. This newsletter contains information on satellite reception for use by educational institutions. 